\title{
The Vasimr Engine: Project Status and Recent Accomplishments
}

\author{
Franklin R. Chang Díaz \\ Advanced Space Propulsion \\ Laboratory, JSC / NASA \\ 13000 Space Center Blvd \\ Houston, TX 77059 \\ (281) $792-5536$ (tel) \\ (281) 792-5661 (fax)
}

F. Wally Baitty

Oak Ridge National

Laboratory

P.O. Box 2009

Oak Ridge, TN

(865)-574-6480 (tel)

(865-576-7926 (fax)

\author{
Jared P. Squire \\ Advanced Space Propulsion \\ Laboratory, JSC / NASA \\ 13000 Space Center Blvd \\ Houston, TX 77059 \\ (281) 792-5673 (tel) \\ (281) 792-5661 (fax)
}

Richard H. Goulding

Oak Ridge National

Laboratory

PO Box 2009

Oak Ridge, TN

(865)-574-6480 (tel)

(865-576-7926 (fax)

\author{
Edgar A. Bering, III \\ University of Houston \\ Departments of Physics and \\ Electrical Engineering \\ Houston, TX 77204-5005 \\ (281) 792-5991 (tel) \\ (713) 743-3543 (tel) \\ (713) 743-3589 (fax) \\ Roger D. Bengtson \\ Physics Department \\ University of Texas at Austin \\ Austin, TX 78712 \\ (512) 471-3943 (tel) \\ (512) 471-8865 (fax)
}

\begin{abstract}
The development of the Variable Specific Impulse Magnetoplasma Rocket (VASIMR) was initiated in the late 1970s to address a critical requirement for fast, high-power interplanetary space transportation. While not being a fusion rocket, it nevertheless borrows heavily from that technology and takes advantage of the natural topology of open-ended magnetic systems. In addition to its high power density and high exhaust velocity, VASIMR is capable of "constant power throttling" a feature, which allows in-flight mission-optimization of thrust and specific impulse to enhance performance and reduce trip time. A NASA-led, research team, involving industry, academia and government facilities is pursuing the development of this concept in the United States. The technology can be validated, in the near term, in venues such as the International Space Station, where it can also serve as both a drag compensation device and a plasma contactor for the orbital facility. Other near-Earth applications in the commercial and scientific satellite sectors are also envisioned. This presentation covers the evolution of the VASIMR concept to its present status, as well as recent accomplishments in our understanding of the physics. Approaches and collaborative programs addressing the major technical challenges will also be presented.
\end{abstract}

\section{INTRODUCTION}

A fundamental problem in human and robotic planetary exploration is the intrinsic limitation of today's chemical rocket. After remarkable advances in the last 50 years, the engineering of these devices has matured to the point were further refinement brings only limited performance gains. While the chemical rocket will continue to provide excellent surface to orbit transportation, new technologies must be called upon to transport humans and cargo in the long journeys to the planets and ultimately the stars.

Plasma rockets on the other hand, open up new and exciting possibilities for fast space transportation. Utilizing ionized gases accelerated by electric and magnetic fields, these devices expand the performance envelope of rocket propulsion far beyond the limits of the chemical rocket. With a properly shaped magnetic duct, the internal energy of a plasma could be extracted in the form of rocket thrust. The duct becomes a magnetic nozzle, the magnetic equivalent of a conventional nozzle. Moreover, the non-physical nature of such a nozzle also suggests an inherent adaptability, which (in analogy to the transmission in an automobile) could continuously tailor the exhaust plume to respond to the conditions of flight. An adaptable nozzle better utilizes the available rocket power, leading to better performance. Although much earlier work identified this benefit, its implementation in chemical rockets with fixed material nozzles was impractical at the time. 
Magnetic mirrors enable the heating of plasmas to tens of millions of degrees by providing an insulating magnetic field between the plasma and its nearest material surface. Because of their open topology, these devices are however inherently leaky, a feature that, while a detriment to controlled fusion, is actually useful in propulsion applications.

The genesis of the Variable Specific Impulse Magnetoplasma Rocket (VASIMR) dates back to the late 1970's. The concept is a natural derivative of mirror machines, and their applications to fusion. A proposed use of VASIMR as primary propulsion for robotic spacecraft is shown in Figure 1.

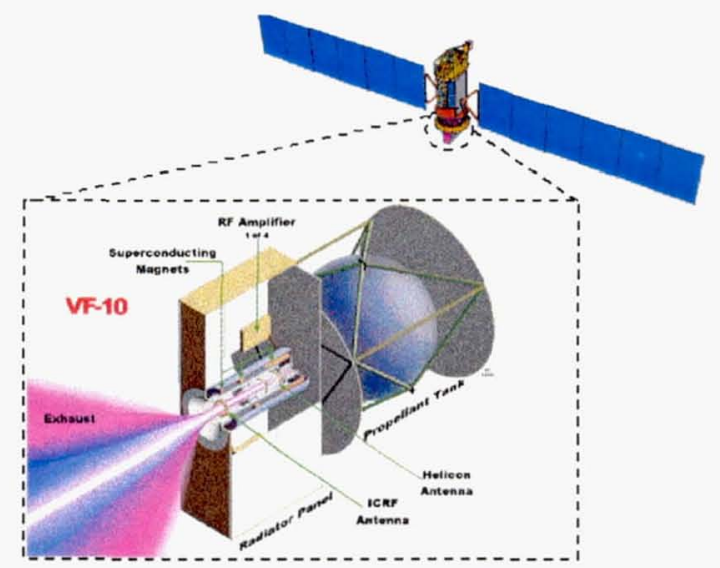

Figure 1: A 10kW VASIMR thruster provides main propulsion capability to the proposed Radiation and Technology Demonstrator (RTD) satellite.

At the present time, the VX-10 experimental device, shown in Figure 2, at the NASA Johnson Space Center in Houston is exploring the physics and engineering of the VASIMR. Similar experiments at the University of Texas at Austin and the Oak Ridge National Laboratory support this research in a collaborative effort involving 7 universities, private industry and two national laboratories.

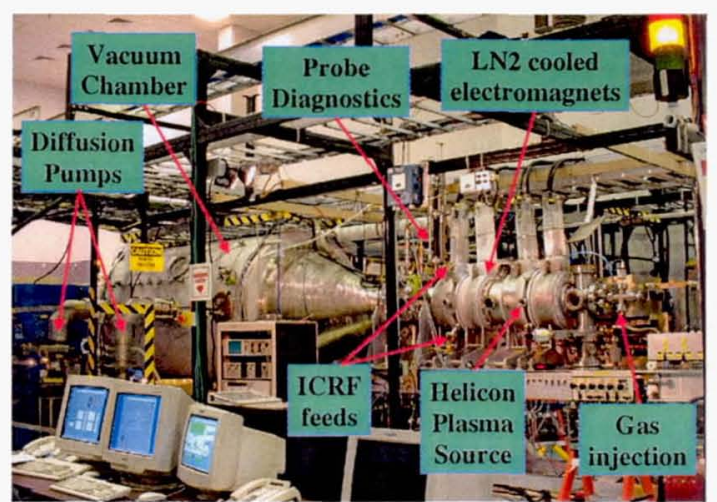

Figure 2: Recent photograph of the VX-10 VASIMR experiment at the Johnson Space Center.

\section{The VASIMR Concept}

The VASIMR consists of three main sections: a helicon plasma source, a radio frequency (RF) power booster, and a magnetic nozzle. Figure 3 shows these three stages integrated with the necessary supporting systems. One key aspect of this concept is its electrodeless design, which makes it suitable for high power density and long component life by reducing plasma erosion and other materials complications. The magnetic field ties the three stages together and, through the magnet assemblies, transmits the exhaust reaction forces that ultimately propel the ship. 


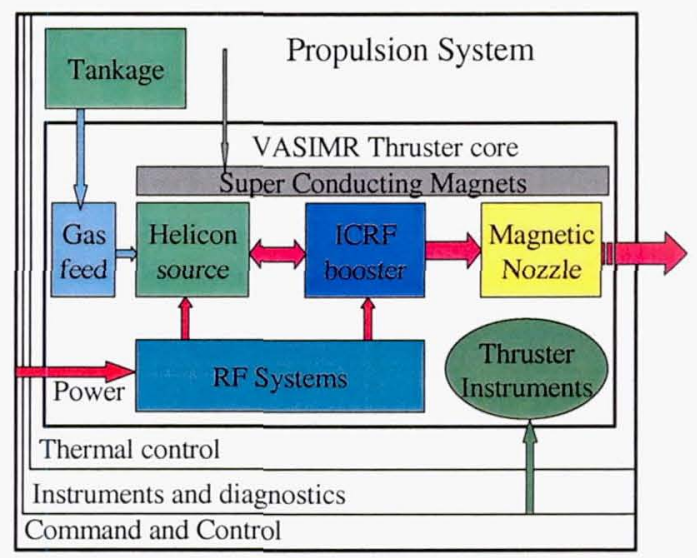

Figure 3: System schematic of the VASIMR

While the three stages must work together, they have distinct functions. The helicon stage handles the main injection of propellant gas and its ionization, the RF booster acts as a power amplifier to further heat the plasma and the magnetic nozzle converts the energy of the fluid into directed flow. VASIMR operates typically with light gases, as the RF booster generally operates near the ion cyclotron resonance frequency; however, the helicon source works effectively with a wide range of gases and gas mixtures, suggesting additional operational modes yet to be explored.

Plasma production by the helicon source must be efficient and optimization of this stage is currently under way. Plasma densities in the $10^{18}$ to $10^{19} / \mathrm{m}^{3}$ range on Helium, Hydrogen, Deuterium, Nitrogen, Argon and other laboratory gases are now routine. The plasma density scales linearly with input power, as shown in Figure 4, with no sign of saturation. As shown in Figure 5, the asymmetric geometry of the magnetic field and the ambipolar potential, driven by the electron temperature, produces a rapid plasma flow.

A large pressure increase in the helicon chamber is also measured during plasma operation, as well as a commensurate downstream force. An interesting observation in the force data of Figure 6 shows an increase in the total force as one samples further downstream in the plume (upper trace is $15 \mathrm{~cm}$. downstream from lower trace.) This behavior is currently under study, but suggests the contribution of the magnetic nozzle to the acceleration of the plasma. The slight decay in the force signal during the pulse is attributed to an increasing neutral background pressure due to pumping limitations.

The RF booster stage, currently under development is targeted to operate at the ion cyclotron resonance frequency, but some manipulation of the magnetic field in the system can also enable experiments at the ion cyclotron $2^{\text {nd }}$ harmonic. Initial experiments have been conducted with a simple dual half-loop antenna configuration. Two separate RF feeds drive two water-cooled copper rings at $90^{\circ}$ phase shift from each other. Important considerations here include the RF antenna loading, which the target plasma provides. For good performance the plasma must pass near the antenna straps and be of sufficient density to couple well to the RF power.

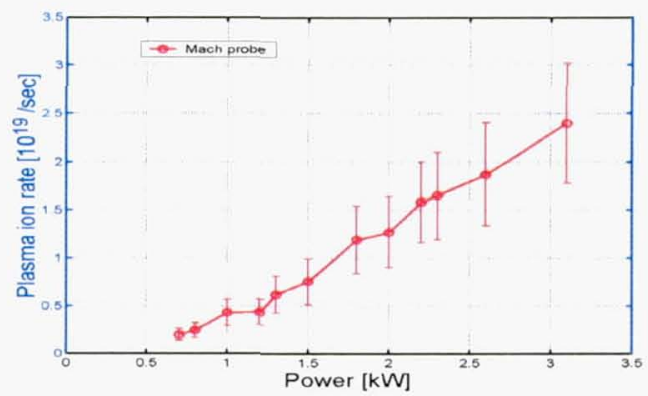

Figure 4: Helicon plasma output vs. power input 


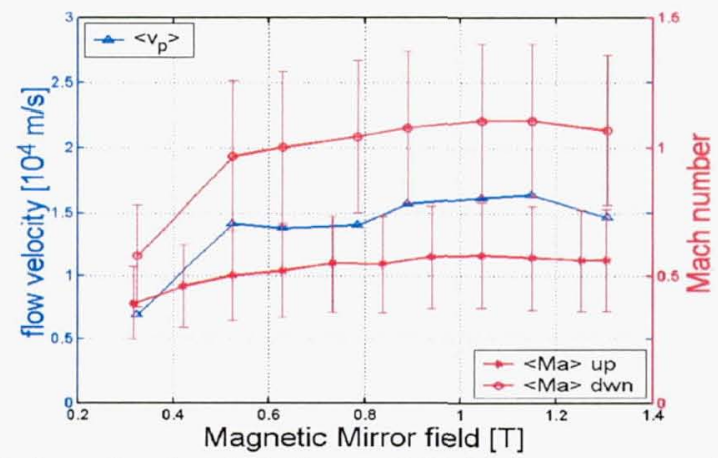

Figure 5: Plasma flow velocity and Mach number data for helium at $3 \mathrm{~kW}$. Measurements are shown upstream and downstream of the magnetic throat.

RF booster heating data are still preliminary; however, interesting and somewhat surprising results have emerged in recent tests. For example, the coupling of RF power at the second harmonic resonance, as shown in Figure 7, is significant. This result has stimulated thinking on other mechanisms, such as ion Bernstein modes, in addition to ion cyclotron resonance, which may be also employed for wave coupling in the booster stage.

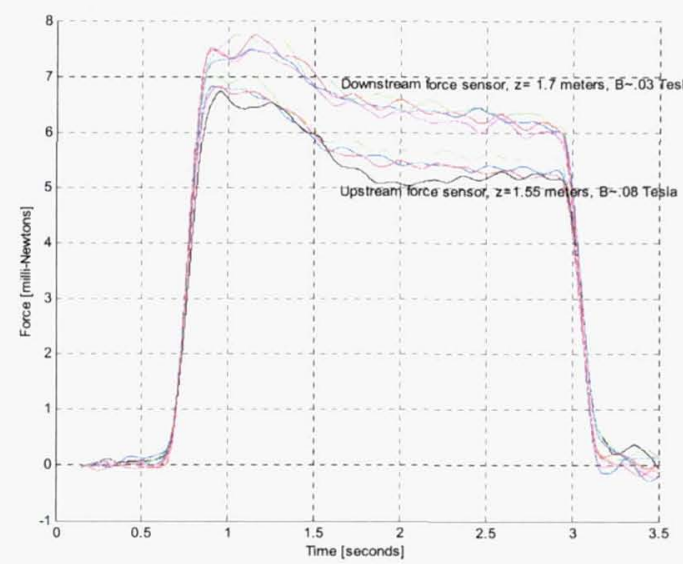

Figure 6: Force sensor data for helium.

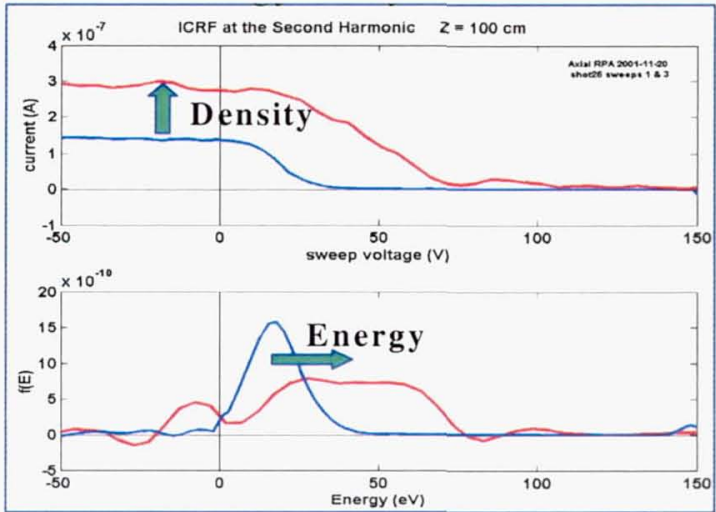

Figure 7: Ion energy data with RF power applied at the $2^{\text {nd }}$ ion cyclotron resonance. Both increases in plasma density and particle energy are observed.

We anticipate that the main thrust of this paper will be to discuss the results of the RF booster experiments that will be carried out in the summer and fall of this year. We have installed a new, larger diameter helicon source, improved RF booster antenna, and other refinements. We will be presenting these results in as much detail as time permits. 